\title{
Modifications to the tetracaine scaffold produce cyclic nucleotide- gated channel blockers with widely varying efficacies
}

Timothy Strassmaier ${ }^{\dagger}$, Ramalinga Uma ${ }^{\dagger}$, Ambarish S. Ghatpande ${ }^{\S}$, Tapasree Bandyopadhyay ${ }^{\dagger}$, Michelle Schaffer", John Witte ${ }^{\|}$, Patrick G. McDougal ${ }^{\|}$, R. Lane Brown ${ }^{\ddagger}$, Jeffrey W. Karpen ${ }^{*},{ }^{\dagger}$

Department of Physiology and Pharmacology ${ }^{\dagger} \&$ Neurological Sciences Institute ${ }^{\ddagger}$, Oregon Health \& Science University, Portland, Oregon 97239; Department of Physiology and Biophysics, University of Colorado Health Sciences Center ${ }^{\S}$, Denver, Colorado, 80262; Department of Chemistry, Reed Collegell, Portland, Oregon, 97202

\section{Supporting Information}

1. Table of HPLC analyses for compounds $2,3,14$ and 15.

2. Table of combustion analyses for compounds $\mathbf{1 4}$ and $\mathbf{1 6}$.

1. Table of HPLC analyses for compounds 2, 3, 14 and 15:

\begin{tabular}{|l|l|l|}
\multicolumn{1}{|c|}{ Compound } & HPLC $1^{*}$ (purity) & \multicolumn{1}{c|}{ HPLC $2^{\dagger}$ (purity) } \\
\hline $\mathbf{2}$ & $99.0 \%$ & $99.0 \%$ \\
$\mathbf{3}$ & $99.3 \%$ & $99.6 \%$ \\
$\mathbf{1 4}$ & $98.2 \%$ & $99.2 \%$ \\
$\mathbf{1 5}$ & $99.9 \%$ & $99.2 \%$ \\
\hline
\end{tabular}

*RP-HPLC: Xterra Prep RP8, 19 x 100 mm, $5 \mu \mathrm{m}$, column (Waters, Milford, MA).

${ }^{\dagger}$ Cation exchange HPLC: PolyCAT A, 21 x 250 mm, $5 \mu \mathrm{m}, 300 \AA ̊$ A column (PolyLC, Southborough, MA).

2. Table of combustion analyses for compounds $\mathbf{1 4}$ and 16:

\begin{tabular}{|c|c|c|c|c|c|c|c|}
\hline & & \multicolumn{3}{|c|}{ Calculated } & \multicolumn{3}{|c|}{ Found } \\
\hline Compd & Formula & $\mathrm{C}$ & $\mathrm{H}$ & $\mathrm{N}$ & $\mathrm{C}$ & $\mathrm{H}$ & $\mathrm{N}$ \\
\hline 14 & $\mathrm{C}_{15} \mathrm{H}_{25} \mathrm{~N}_{3} \mathrm{O}_{2} \cdot 2 \mathrm{CH}_{3} \mathrm{COOH}$ & 57.12 & 8.33 & 10.52 & 56.15 & 8.90 & 11.60 \\
\hline 16 & $\mathrm{C}_{11} \mathrm{H}_{16} \mathrm{~N}_{2} \mathrm{O}_{2}$ & 62.74 & 7.85 & 13.17 & 63.44 & 7.74 & 13.45 \\
\hline
\end{tabular}

